\title{
Synthesis of specific fatty acids contributes to VLDL-triacylglycerol composition in humans with and without type 2 diabetes
}

\author{
M. S. Wilke • M. A. French • Y. K. Goh • E. A. Ryan • \\ P. J. Jones • M. T. Clandinin
}

Received: 11 March 2009/Accepted: 30 April 2009/Published online: 18 June 2009

(C) Springer-Verlag 2009

\begin{abstract}
Aims/hypothesis It is recommended that patients with diabetes reduce their intake of saturated fat and increase their intake of monounsaturated fat or carbohydrate. However, high-carbohydrate diets may result in higher saturated fatty acids in VLDL-triacylglycerol. This is attributed to de novo lipogenesis, although synthesis of specific fatty acids is rarely measured. The objective of this study was to examine the contribution of de novo fatty acid synthesis to VLDL-triacylglycerol composition. It was hypothesised that levels of total and de novo synthesised fatty acids would increase with increased carbohydrate intake in diabetic participants.

Methods Seven individuals with type 2 diabetes mellitus and seven matched non-diabetic controls consumed two diets differing in fat energy (lower fat $<25 \%$, higher fat $>35 \%$ ) for 3 days in a randomised crossover design. Blood samples were drawn before and $24 \mathrm{~h}$ after the ingestion of ${ }^{2} \mathrm{H}$-labelled water.
\end{abstract}

Electronic supplementary material The online version of this article (doi:10.1007/s00125-009-1405-9) contains supplementary material, which is available to authorised users.

M. S. Wilke • M. A. French • Y. K. Goh • M. T. Clandinin $(\bowtie)$

Alberta Institute for Human Nutrition, University of Alberta,

4-10 Agriculture-Forestry Centre,

Edmonton, AB, Canada, T6G 2P5

e-mail: tclandin@ualberta.ca

E. A. Ryan $\cdot$ M. T. Clandinin

Department of Medicine, University of Alberta,

Edmonton, AB, Canada

P. J. Jones

Richardson Centre for Functional Foods and Nutraceuticals,

University of Manitoba,

Winnipeg, MB, Canada
Results In the control participants, the higher-fat diet resulted in a $40 \%$ reduction in VLDL-triacylglycerol fatty acids because of decreases in myristic, palmitic, palmitoleic and linoleic acids, but the opposite trend occurred in participants with diabetes. The lower-fat diet increased the fractional synthesis rate by $35 \%$ and $25 \%$ in the control and diabetes participants, respectively (range: $0-33 \%$ ). Palmitate accounted for $71 \%$ of fatty acids synthesised (range: 44 $84 \%$ total de novo synthesised fatty acids).

Conclusions/interpretation ${ }^{2} \mathrm{H}$ incorporation was used for the first time in humans showing variability in the synthesis rate of specific fatty acids, even palmitic acid. A lower-fat diet stimulated saturated fatty acid synthesis at high rates, but no net stimulation of synthesis of any fatty acid occurred in the diabetes group. The implications of this finding for our understanding of lipid metabolism in diabetes require further investigation.

Keywords De novo lipogenesis - Deuterium incorporation · Dietary carbohydrate - Dietary fat $\cdot$ Fat metabolism .

Fatty acid composition · Mass spectrometry .

Plasma triacylglycerols · Type 2 diabetes mellitus · VLDL

$\begin{array}{ll}\text { Abbreviations } \\ \text { 14:0 } & \text { Myristate } \\ \text { 16:0 } & \text { Palmitate } \\ \text { 16:1 } & \text { Palmitoleate } \\ 18: 0 & \text { Stearate } \\ 18: 1 & \text { Oleate } \\ 18: 2 & \text { Linoleate } \\ 18: 3 & \alpha \text {-Linolenate } \\ \text { CVD } & \text { Cardiovascular disease } \\ \text { DNFAn } & \text { Net de novo synthesised fatty acids } \\ \text { DNFAr } & \text { Relative de novo synthesised fatty acids } \\ \text { DNL } & \text { De novo lipogenesis }\end{array}$


FA Fatty acid

FAMEs Fatty acid methyl esters

GC/P/IRMS Gas chromatography/pyrolysis/isotope ratio mass spectrometry

$\mathrm{HF}$

LF

$\mathrm{P}: \mathrm{S}$

TG

High-fat/low-carbohydrate diet

Low-fat/high-carbohydrate diet

Polyunsaturated to saturated fatty acid ratio

Triacylglycerol

\section{Introduction}

Diabetes management focuses on glucose control. However, perturbations in lipid metabolism are connected to insulin resistance and atherogenesis development. Glucose and lipid metabolism are not independent but connected via de novo lipogenesis (DNL), the conversion of glucose to fatty acids (FAs) and triacylglycerol (TG) by the liver. Diet is important in managing glucose concentrations and risk of cardiovascular disease (CVD) in patients with diabetes. Reducing dietary saturated fat is the most common recommendation to reduce CVD risk, even though low-fat/high-carbohydrate diets increase fasting plasma TG concentrations [1], an independent risk factor for CVD [2]. Compared with higherfat intake, low-fat/high-carbohydrate diets change the FA composition of plasma lipoproteins and red cells, which is often attributed to DNL of non-essential FAs [3-5]. Synthesis of specific FAs is rarely measured because of limited technological capabilities.

The importance of dietary fat composition as a determinant of serum and adipose tissue FA composition has been investigated [6, 7]. Quantity of dietary fat has recently been considered with regard to the composition of circulating FAs [3-5], particularly saturated FAs. Like dietary fat 'quality', serum FA 'quality' may be important in the longterm development of metabolic disease [8]. FA composition is thought to influence metabolic processes through effects on insulin action [9] and lipid metabolism [10], which are altered in individuals with diabetes.

VLDL is a major carrier of TGs. Sources of FAs for hepatic TG synthesis are DNL, chylomicron remnant TG and plasma NEFAs, which vary depending on diet, feeding [11] and metabolic state [12]. Chylomicron-TG and adipose-TG FA composition reflect that of diet, but VLDL-TG differs [13]. It is thought that FAs from exogenous and endogenous sources may be sequestered into cytosolic TG stores before incorporation into VLDLTG [14], resulting in an FA profile that differs from dietary composition. Thus, endogenous contribution of FAs from DNL to VLDL-TG may be a major factor. Palmitate is the major quantitative product of hepatic DNL and increases when DNL is stimulated by high carbohydrate intake,

whereas linoleate decreases in VLDL-TG [15]. DNL is variable but contributes from $2 \%$ to $30 \%$, and is upregulated by carbohydrate intake and hyperinsulinaemia [16] High-carbohydrate diets show a consistent effect of inducing both DNL and hypertriacylglycerolaemia [15, 17]. Hypertriacylglycerolaemia may be prevented by a higher dietary fat concentration $[1,15]$ and guidelines recognise that higher fat intake (i.e. $\geq 30 \%$ energy intake) may be acceptable if primarily composed of mono- and polyunsaturated fats and low in trans FAs [18].

If DNL contributes saturated FAs to VLDL-TG, this could have important metabolic effects. It is not known if synthesis of specific FAs varies between individuals, diets and in metabolic states such as type 2 diabetes. The current study estimates synthesis of individual FAs for the first time using ${ }^{2} \mathrm{H}$-incorporation techniques. The objective was to determine if DNL contributes to VLDL-TG FA composition and if hepatic FA synthesis varies depending on the presence of diabetes and on dietary carbohydrate and fat content. It was hypothesised that DNL of total and saturated FAs is higher in diabetic individuals compared with a matched control group, particularly following lower fat/ higher carbohydrate intake, resulting in differences in VLDL-TG FA composition.

\section{Methods}

Study participants Eleven individuals with type 2 diabetes and ten non-diabetic individuals were recruited, with a total of seven in each group completing the study. Participants were recruited through the outpatient Metabolic Clinic at University of Alberta Hospitals, Capital Health Authority Diabetes Registry and a database of volunteers compiled from respondents to another study. All participants provided informed consent. The study was approved by the Human Research Ethics board at the University of Alberta.

Baseline screening results are shown (Table 1). Volunteers were excluded if they had a fasting TG concentration $>4.0 \mathrm{mmol} / \mathrm{l}$, were taking lipid-lowering drugs or were not in reasonable metabolic control. Participants using other medications, including metformin and levothyroxine, were instructed to continue and record their normal medication. Test results and medications were reviewed by an endocrinologist.

Study design Participants were examined after two feeding periods comprising 3 days each in a crossover design and 1 month washout period. Participants picked up packaged meals and were instructed to consume only food and beverages provided (except energy-free fluids), exclude alcohol intake and not to exercise beyond a moderate amount (explained in detail). Unwashed food containers were returned as an indication of compliance. Participants 
Table 1 Baseline characteristics of group with type 2 diabetes and matched (sex, age, BMI) non-diabetic control group completing the study

Values are mean $\pm \mathrm{SD}$

Baseline measurements were taken a minimum of 1 month before beginning the study protocol

*Significant difference from control group, $p<0.05$

ApoE, apolipoprotein E; F, female; $M$, male

\begin{tabular}{lll}
\hline Characteristic & Control group & Diabetes group \\
\hline Sex (M/F) & $3 / 4$ & $3 / 4$ \\
Age (years) & $51.4 \pm 9.2$ & $50.0 \pm 8.8$ \\
BMI (kg/m $\left.{ }^{2}\right)$ & $33.5 \pm 8.3$ & $33.2 \pm 7.5$ \\
Weight (kg) & $93.4 \pm 24.1$ & $91.9 \pm 15.4$ \\
Waist (cm) & $105.6 \pm 15.8$ & $105.6 \pm 13.5$ \\
Alkaline phosphatase (U/l) & $77.0 \pm 26.9$ & $72 \pm 16.7$ \\
Alanine aminotransferase (U/l) & $25.0 \pm 12.2$ & $29.4 \pm 14.0$ \\
Creatinine ( $\mu m o l / 1)$ & $83.1 \pm 18.6$ & $70.3 \pm 15.3$ \\
Glucose (mmol/l) & $5.0 \pm 0.4$ & $6.2 \pm 1.1^{*}$ \\
HbA 1 (\%) & $5.3 \pm 0.4$ & $5.9 \pm 0.5^{*}$ \\
Insulin (pmol/l) & $69 \pm 53$ & $95 \pm 55$ \\
C-peptide (nmol/l) & $1.1 \pm 0.4$ & $1.5 \pm 0.5$ \\
HOMA & $2.22 \pm 1.7$ & $3.83 \pm 2.4$ \\
NEFA (mmol/l) & $0.88 \pm 0.4$ & $0.66 \pm 0.3$ \\
Triacylglycerols (mmol/l) & $1.35 \pm 0.4$ & $1.96 \pm 0.8$ \\
Total cholesterol (mmol/l) & $5.41 \pm 0.8$ & $4.76 \pm 0.6$ \\
LDL-cholesterol (mmol/l) & $3.46 \pm 0.7$ & $2.67 \pm 0.6^{*}$ \\
HDL-cholesterol (mmol/l) & $1.34 \pm 0.2$ & $1.21 \pm 0.2$ \\
Cholesterol:HDL & $4.09 \pm 0.6$ & $4.01 \pm 0.6$ \\
ApoE genotype (E2/E3, E3/E3, E3/E4, E4/E4) & $3,3,0,1$ & $2,3,2,0$ \\
\hline & &
\end{tabular}

were tested at the same time each month to account for hormonal variation in the menstrual cycle.

On test day 1 (diet day 3 ) of each diet treatment, fasting blood samples were obtained following a $12 \mathrm{~h}$ overnight fast and analysed for 'background' ${ }^{2} \mathrm{H}$ enrichment. Subsequently, participants drank a priming dose of ${ }^{2} \mathrm{H}_{2} \mathrm{O}$ at $1.0 \mathrm{~g} / \mathrm{kg}$ estimated body water ( $60 \%$ of body weight) to rapidly increase plasma ${ }^{2} \mathrm{H}$ concentrations. At regular intervals throughout the next $24 \mathrm{~h}$, a maintenance dose of $1.0 \mathrm{~g}$ ${ }^{2} \mathrm{H}_{2} \mathrm{O} / \mathrm{kg}$ estimated body water diluted in 1.51 bottled water was ingested to maintain plasma ${ }^{2} \mathrm{H}$ concentrations at plateau. Breakfast was served immediately and remaining meals were to be consumed a minimum of $12 \mathrm{~h}$ before the final blood draw. The final $12 \mathrm{~h}$ fasting blood sample was drawn on test day 2 at 09:00 hours and was used for determinations of fasting VLDL-TG FA concentrations and $24 \mathrm{~h}$ FA synthesis. Total plasma lipid, glucose and insulin concentrations were analysed using automated enzymatic procedures.
Diet composition Diets were formulated to meet the participant's energy needs using the Harris Benedict equation and an activity factor. The high-carbohydrate/ low-fat diet (LF) consisted of $<25 \%$ of energy from fat and included complex carbohydrates with a low glycaemic index. The high-fat/low-carbohydrate diet (HF) consisted of the same menu items as the LF (Table 2) with isoenergetic addition of canola oil to achieve $\sim 35 \%$ of energy from fat. The aim was to increase the intake of monounsaturated fat but not that of saturated or polyunsaturated fat, which should be limited to $<7 \%$ and $<10 \%$ of total energy intake, respectively [18]. Safflower and flax oils were added to LF meals to increase the polyunsaturated fat energy contributed by canola oil to HF. Meals and diets were similar in energy and macronutrient composition and identical meals were consumed on all 3 days. Feeding of participants was controlled for intake, blinding and randomisation of diet treatment order.

Table 2 Menu items consumed during lower- and higher-fat diets

\begin{tabular}{lll}
\hline Breakfast & Lunch & Dinner \\
\hline Orange juice & Wholewheat pasta & Turkey sandwich \\
Blueberry oatbran muffins & Tomato-based pasta sauce (vegetables, beef, mozzarella) & Raspberry or fig newtons ${ }^{\mathrm{a}}$ \\
Scrambled egg & Peas & Melon pieces \\
Bread & Apple & \\
\hline
\end{tabular}

${ }^{\text {a }}$ Also known as raspberry or fig rolls. Meals were identical on all 3 days of diet intervention for both higher- and lower-fat diets. Safflower oil, flax oil and margarine with $50 \%$ less fat were added to the lower-fat menu and canola oil and canola margarine added to the higher-fat menu 
Diet composition (Table 2) was calculated using Food Processor II nutrient analysis software (V9.6.2, Esha Research, Salem, OR, USA), which included integrated FA profiles from GC analysis of added oils/spreads (not shown). Fat from test meals was extracted and analysed by GC to confirm FA composition.

Determination of VLDL-TG FA composition by gas liquid chromatography Blood was centrifuged and plasma immediately extracted and refrigerated. Extraction of VLDL-TG FAs involved ultracentrifugation of plasma to separate out chylomicrons within $24 \mathrm{~h}$ of collection. The VLDL portion was extracted by non-equilibrium density-gradient ultracentrifugation [19]. TGs were removed [6] using a Folch extraction, followed by addition of internal standard (17:0), separation by TLC and quantitative recovery of the TG fraction [20]. Following methylation, fatty acid methyl esters (FAMEs) were identified and quantified by GC. Methyl esters of all saturated, cis-monounsaturated and cispolyunsaturated FAs from 14 to 24 carbons in chain length were analysed; however, only major FAs (14-18 carbons) were included in the results. The proportions of 20-24 carbon FAs in the VLDL-TG comprised $<4 \%$.

Estimation of fatty acid synthesis by ${ }^{2} \mathrm{H}$ incorporation Isotopic analysis of ${ }^{2} \mathrm{H}$ enrichment in VLDL-TG FAs was performed by ${ }^{2} \mathrm{H}$ incorporation techniques using isotoperatio MS. The technique is similar to that used by Konrad et al. [17]; however, combustion of FAMEs to $\mathrm{H}_{2}$ or $\mathrm{H}^{2} \mathrm{H}$ before injection into the isotope-ratio mass spectrometer (IRMS) was not required. FAME samples were injected into a GC and effluent was pyrolysed in a furnace at $1,450^{\circ} \mathrm{C}$ to form $\mathrm{H}_{2}$ or $\mathrm{H}^{2} \mathrm{H}$, carbon monoxide and water. $\mathrm{CO}$ and $\mathrm{H}_{2} \mathrm{O}$ were removed and the $\mathrm{H}_{2} / \mathrm{H}^{2} \mathrm{H}$ mixture passed into the IRMS where enrichment of ${ }^{2} \mathrm{H}$ in each peak from the GC/pyrolysis/ IRMS (GC/P/IRMS) was measured (ThermoFinnigan Delta PlusXL; Breman, Germany).

The method of Konrad et al. quantifies total FA synthesis whereas the GC/P/IRMS method permits quantitative and qualitative determination of individual FAs and atom \% enrichment of ${ }^{2} \mathrm{H}$ present in individual FAMEs (see Electronic supplementary material [ESM]). Instrument sensitivity is $10 \mathrm{nmol}$ on columns with an internal precision of 0.25 per mil (\%o). All samples were run in duplicate; however, if the results were not consistent, samples were reprepared and rerun in duplicate. If the area under the peak for a particular FA was $<0.1$, the concentration of the FA for the purposes of ${ }^{2} \mathrm{H}$ enrichment calculations was considered negligible. Peaks for 18:1 $n^{-7}$ and 18:1 $n^{-9}$ isomers were considered as total 18:1.

Plasma water ${ }^{2} \mathrm{H}$ enrichment $\mathrm{It}$ is assumed when using the ${ }^{2} \mathrm{H}$ incorporation method that all cell membranes are permeable to ${ }^{2} \mathrm{H}_{2} \mathrm{O}$ and that plasma ${ }^{2} \mathrm{H}$ enrichment is identical to that of the intracellular pool from which the VLDL-TG incorporates ${ }^{2} \mathrm{H}$ [21]. Isotopic analysis of plasma water ${ }^{2} \mathrm{H}$ was therefore measured as 'precursor' enrichment. Standard vacuum techniques were performed [21] using a 903D dual-inlet IRMS (VG Isogas, Middlewich, UK). The dilution space of each participant was obtained from plasma ${ }^{2} \mathrm{H}$ enrichments [22].

Statistical methods Statistical analyses and graphs were performed and prepared using Statistica software (StatSoft, Tulsa, USA) and SAS software 8.0 (SAS Institute, Cary, NC, USA). GraphPAD Prism (V5.0, GraphPAD Software, San Diego, USA) was used to prepare most of the figures. Paired $t$ tests compared the effect of LF or HF within each group. Wilcoxon matched pair $t$ tests compared differences between groups. Repeated measures two-factor ANOVA with Bonferroni post tests were used for main effects. Sample size calculations based on previous results expecting a decrease in plasma TG concentration of $25 \%$ estimated completion of seven participants as sufficient statistical power to achieve significance. All relationships between variables were tested using Spearman's rank correlation for non-parametric data. Statistical significance was set at a $p$ value $<0.05$.

\section{Results}

Of the 21 participants recruited, 14 completed both diets and were included in the results ( $n=7 /$ group, Table 1$)$. The major obstacle to recruitment was exclusion of respondents taking lipid-lowering medications. Of the participants who dropped out, four withdrew at the outset, two after completing only one diet period and one because of illness. Diabetic and non-diabetic control groups were similar in characteristics except plasma glucose, $\mathrm{HbA}_{1 \mathrm{c}}$ and LDLcholesterol concentrations (Table 1).

Dietary analyses Three participants included in the results did not consume all food in the first diet as prescribed. Uneaten food was weighed and the equivalent energy amount of the same item was subtracted from the second diet for these participants. All items from the second diet were consumed in their entirety by all participants. Upon study completion, a questionnaire was administered to estimate dietary blinding success. Nine out of 14 participants were unable to correctly discriminate diets, four identified both diets correctly and one questionnaire was unreturned.

Clinical measures Glucose was significantly higher in the diabetes group following the LF (Fig. 1a). No other differences were found between diabetes and control groups 

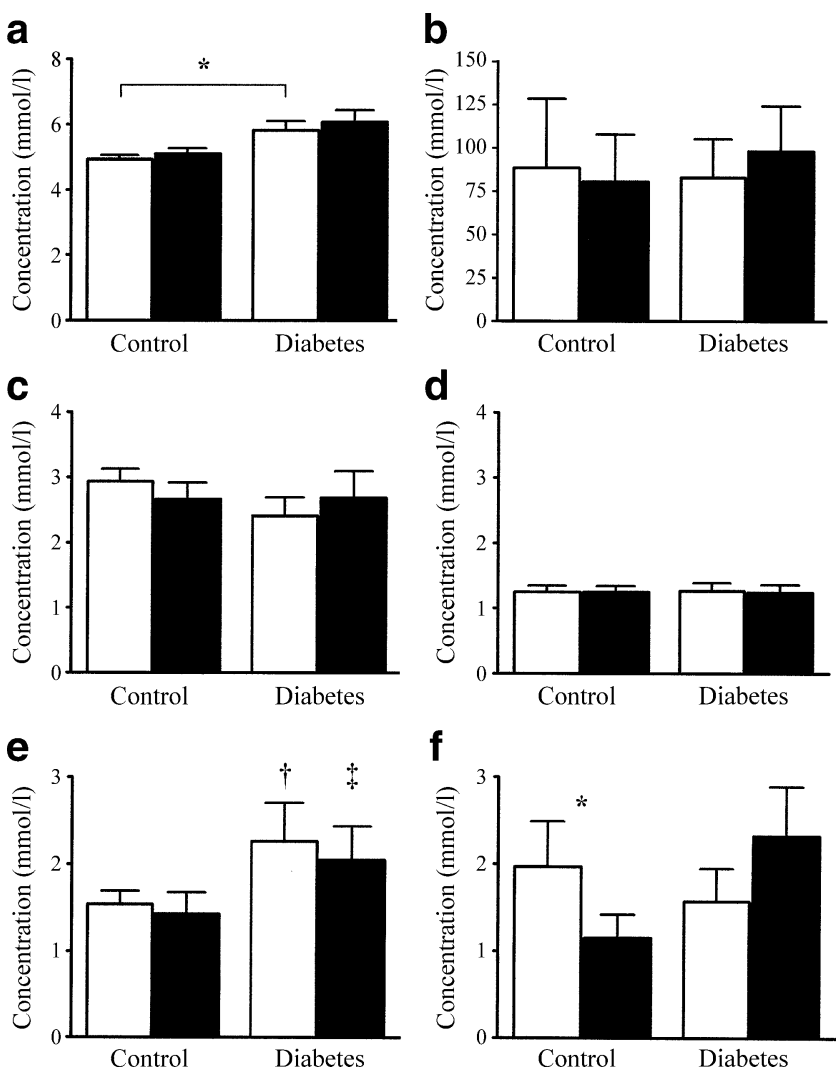

Fig. 1 Fasting clinical and lipid variables. (a) Glucose, (b) insulin, (c) LDL-cholesterol, (d) HDL-cholesterol, (e) triacylglycerols and (f) VLDL-TG fatty acid concentrations were measured in control and diabetic participants following 3 days of lower (white bars) and higher (black bars) fat intake. Values are means with SEM error bars. A significant difference at ${ }^{*} p<0.05$ as determined by paired $t$ tests (within groups) or Wilcoxon matched pair $t$ tests (between groups). A main effect of diabetes is denoted by ${ }^{\dagger} p<0.5$ and ${ }^{\star} p<0.01$

or between diets for any clinical measure (Fig. 1b-d), except TG concentration. A main effect of diabetes indicated that participants with diabetes had higher TG following both LF $(p<0.05)$ and HF $(p<0.01)$ (Fig. 1e).

Total VLDL triacylglycerol fatty acid concentration and synthesis

The control group had a lower fasting VLDL-TG FA concentration after 3 days of HF than after LF $(p=0.03)$ (Fig. 1f). The concentration of FA in VLDL-TG ranged from 1.2 to 3.9 and 1.2 to $3.8 \mathrm{mmol} / \mathrm{l}$ for diabetic participants and 0.8 to 2.0 and 0.6 to $2.7 \mathrm{mmol} / 1$ for controls following $\mathrm{LF}$ and HF, respectively.

A significant effect of diet is shown for fractional rate of DNL of FA in VLDL-TG (DNFAr [ESM]) (Fig. 2a) after an overnight $12 \mathrm{~h}$ fast but not net synthesis (DNFAn [ESM]) (Fig. 2b). The relative synthesis rate was significantly lower following HF than following LF and ranged from 3\% to $33 \%$ and $5 \%$ to $25 \%$ in control participants and from $2 \%$ to $28 \%$ and 0 to $25 \%$ in diabetic participants following LF and $\mathrm{HF}$, respectively.

Individual VLDL-triacylglycerol fatty acid concentration and synthesis

The HF resulted in lower concentrations of most FAs in VLDL-TG in control participants (Fig. 3a), but not in the diabetes group (Fig. 3b). Myristate (14:0) $(p=0.018)$, palmitate $(16: 0)(p=0.013)$, palmitoleate $(16: 1)(p=0.018)$ and linoleate $(18: 2)(p=0.024)$ were significantly reduced following HF in control participants. The concentrations of stearate (18:0), oleate (18:1) and $\alpha$-linolenate (18:3) in VLDL-TG were not significantly changed after 3 days of HF. Average palmitate and oleate concentrations in diabetic participants were almost twofold higher than those in control participants $(p=0.078)$. The ranges of concentration were: $0.1-0.8$ and $0.08-1.2 \mathrm{mmol} / \mathrm{l}$ for palmitate and $0.2-$ 1.1 and $0.2-2.1 \mathrm{mmol} / 1$ for oleate in control and diabetic participants, respectively.

No differences were found between groups or diets for DNFAr (Fig. 3c, d) or DNFAn [ESM] (Fig. 3e, f) of any FA. Figure 4 shows the concentrations of de novo synthesised FAs in VLDL-TG for each participant. If an FA is not shown, the concentration was too low to reliably detect ${ }^{2} \mathrm{H}$ by the IRMS.

Relationships between clinical measures, VLDL-TGFA concentration and synthesis When the data were considered as one group or separated into diabetes and control groups, no correlations were found between FA synthesis and fasting plasma TGs. When correlations were tested for the entire group, there was a relationship between BMI and synthesis rates of palmitate $(r=0.68, p=0.007)$, palmitoleate $(r=0.71, p=0.004)$ and total FA $(r=0.71, p=0.005)$ after $\mathrm{HF}$, and BMI with palmitate $(r=0.53, p=0.05)$ and total FA a

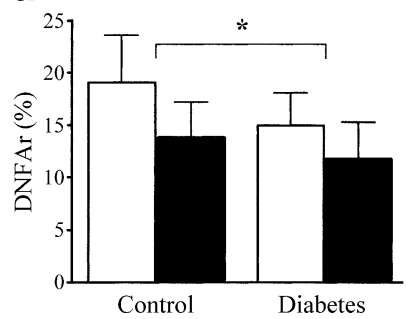

b

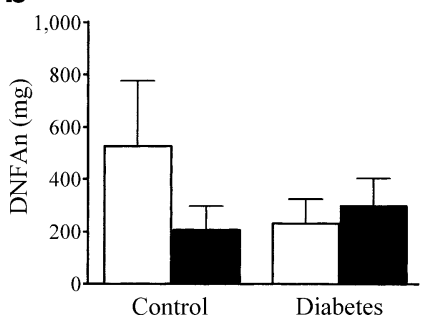

Fig. 2 Fasting total fatty acid synthesis in VLDL-triacylglycerol. (a) Relative and (b) net de novo fatty acids were measured in control and diabetic participants following 3 days of lower (white bars) and higher (black bars) fat intake. Values are means with SEM error bars. A significant main effect of diet at $* p<0.05$ as determined by repeated measures ANOVA. No other significant differences were detected 
Fig. 3 Fasting VLDL-

triacylglycerol fatty acid variables. (a, b) Concentration, (c, d) relative synthesis (DNFAr) and (e, f) net synthesis (DNFAn) of individual fatty acids in VLDLTG of control $(\mathbf{a}, \mathbf{c}, \mathbf{e})$ and diabetic $(\mathbf{b}, \mathbf{d}, \mathbf{f})$ participants following lower (white bars) or higher (black bars) fat intake for 3 days. Values are means with SEM error bars. A significant difference between LF and HF within groups (paired $t$ test) is denoted by $* p<0.05$. No other main effects or differences between diets or groups was detected. For diets and groups $n=7$, with the exceptions: $n=4$ for 14:0 higher fat; for the diabetes group 18:0 higher fat $n=6$; control group 18:0 higher fat $n=5$; and lower fat $n=6$ for relative and net synthesis
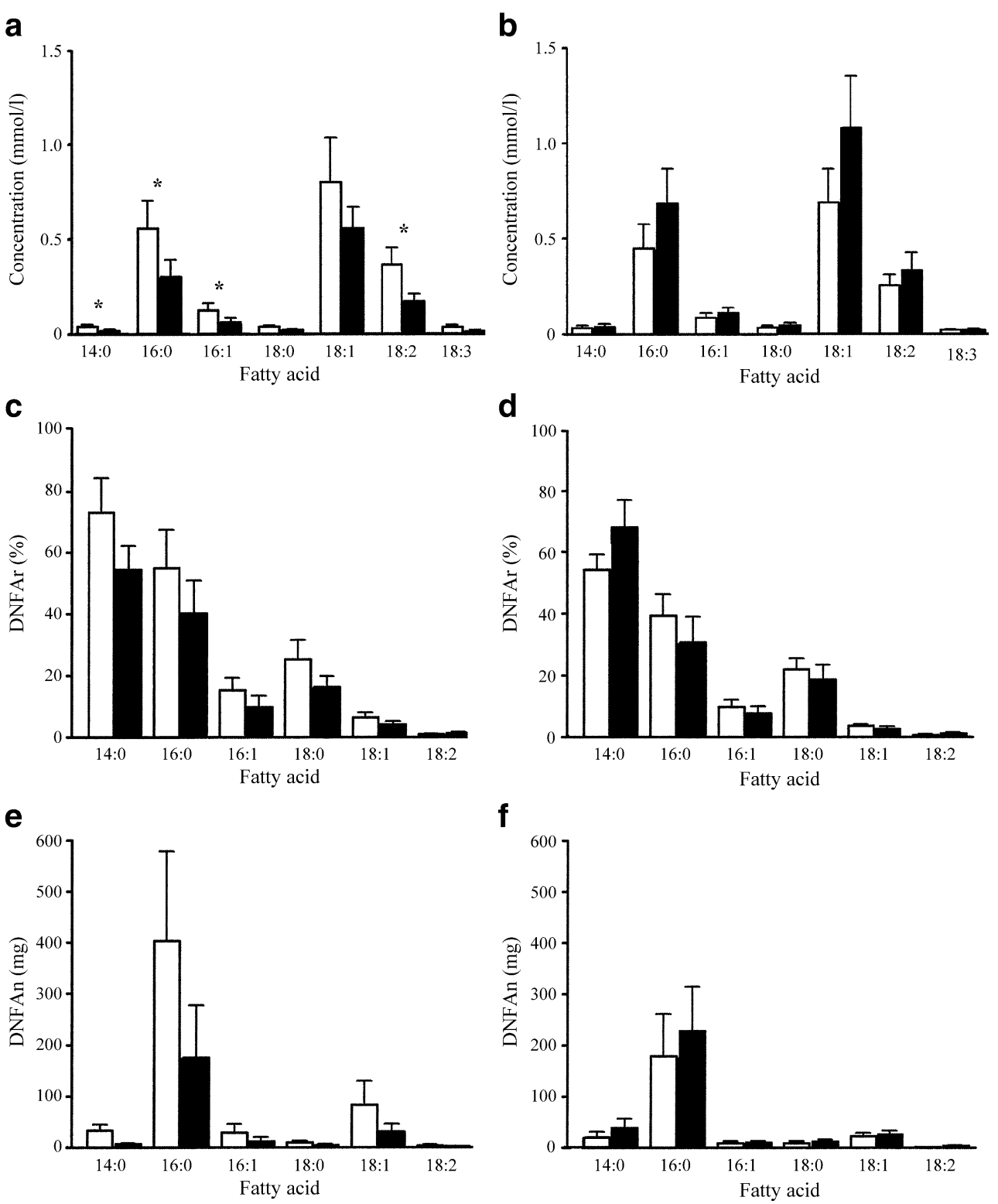

f

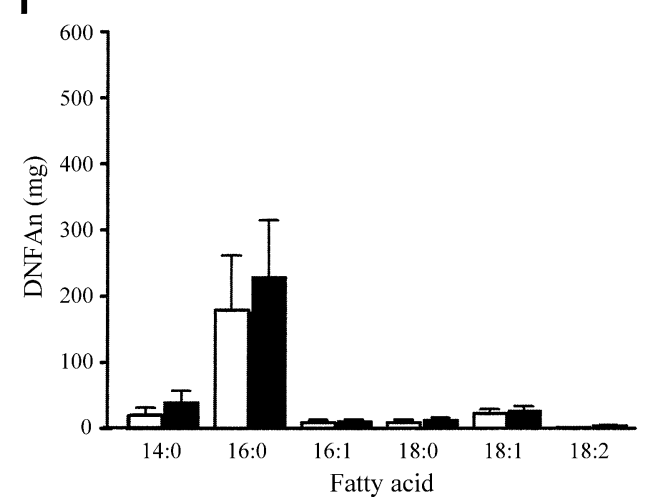

$(r=0.62, p=0.02)$ synthesis rates after LF. Although total synthesis rates were not correlated with glucose concentrations, correlations were found with HOMA (LF: $r=0.65$, $p=0.01$; HF: $r=0.60, p=0.02$ ) and with insulin concentrations (LF: $r=0.68, p=0.008$; HF: $r=0.58, p=0.03$ ). These relationships were weaker following $\mathrm{HF}$ intake. HOMA correlated with fasting VLDL-TG myristate concentration following both $\operatorname{LF}(r=0.53, p=0.05)$ and $\mathrm{HF}$ $(r=0.70, p=0.005)$. When separated into control and diabetes groups, this relationship remained for the control group after LF $(r=0.82, p=0.023)$ and the diabetes group after HF $(r=0.98, p<0.0001)$.

Relationships between total VLDL-TG FA concentration and the relative rate of FA synthesis also depended on the diet consumed. Following the HF diet, only myristate and palmitate concentrations in VLDL-TG correlated with the rate of palmitate synthesis $(r=0.58, p=0.03$ and $r=0.55$, $p=0.04$, respectively). For $\mathrm{LF}$, the strongest relationship found was a correlation between the concentration of myristate in VLDL-TG and the synthesis rate of myristate $(r=0.79, p=0.0008)$. VLDL-TG myristate concentration was also correlated with synthesis rates of palmitate $(r=0.66, p=0.01)$ and palmitoleate $(r=0.76, p=0.002)$.

\section{Discussion}

Research surrounding the FA composition of DNL and circulating TGs is not commonly undertaken. In the present study, considerable variation between VLDL-TG FA concentrations, the types of FA present and hepatic synthesis of FAs was observed when participants consumed the same 


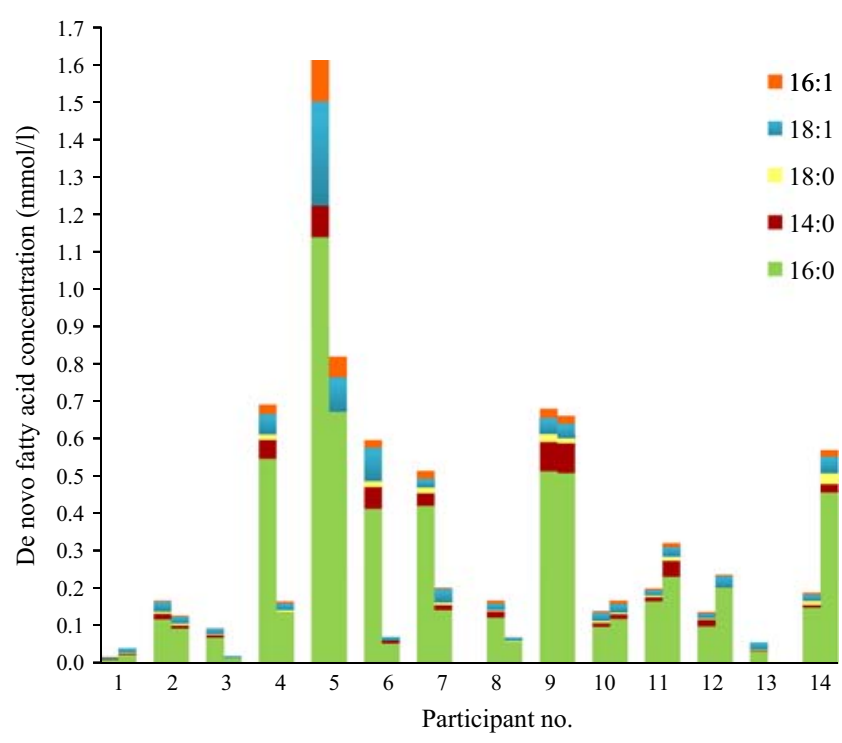

Fig. 4 Concentrations of de novo fatty acids for individual participants. The concentrations of de novo fatty acids synthesised in VLDL-TG for control participants (1-7) and matching diabetic participants (8-14) following 3 days of lower-fat (left bar of each pair) and higher-fat (right bar of each pair) diets

diet. Diabetes and diet seemed to have unique effects on FA metabolism. The LF diet distinctly stimulated lipogenesis, but affected VLDL-TG FA differently in diabetic and nondiabetic participants.

The experimental diets were designed so that the oleate comprised most of the difference in fat intake. VLDL-TG oleate concentration was unchanged following HF intake, even though the dietary amount of this FA was twice that in the LF. A decrease in all VLDL-TG FAs, particularly saturated FAs, occurred in control participants after HF, despite being $>35 \%$ fat energy vs $<25 \%$ in the LF. This reduction in VLDL-TG after 3 days of HF intake was consistent with a significant reduction in total plasma $\mathrm{TG}$ concentration. When VLDL was examined to determine which FAs contributed to this difference, the average values for all FAs measured had decreased after HF, including a significant effect of diet on myristic, palmitic and palmitoleic acids. Linoleic acid was also lower in control participants after HF compared with LF, which was unexpected considering the results with lower-fat/higher-carbohydrate diets in other studies. Previous research involving carbohydrate overfeeding [23] or very-low-carbohydrate diets [3] indicated that high carbohydrate intake results in higher concentrations of palmitate and lower linoleic acid in serum lipids, often in disproportion to the levels provided by diet. More moderate changes in total dietary fat intake $(20 \%$ vs $45 \%$ of energy from fat) also seem to result in changes in FA composition, with lower fat intakes resulting in a lower proportion of linoleic acid in plasma FAs and a higher proportion of saturated and $n-3$ FAs [5]. Diets fed in many studies were longer term and/or involved greater macronutrient manipulation than in the current study. Although diets were matched in the proportion of energy from linoleic acid (Table 3), the ratio of polyunsaturated to saturated fatty acids (P:S) was high in the LF of this study and may have compensated for any reductions expected to occur by increasing carbohydrate intake.

The response of VLDL-TG FA to diet observed in controls was not evident in diabetic participants regarding amount or composition (Fig. 1e, f). The differences
Table 3 Comparison of the composition of the $8,792 \mathrm{~kJ}$ diet as computer formulated and as GC analysis of fatty acid composition

\footnotetext{
${ }^{\mathrm{a}}$ The average energy intake was $8,792 \mathrm{~kJ}$ as calculated by Food Processor II nutrient analysis software (V7.4, Esha Research, Salem, OR, USA) after the fatty acid constituents of added oils (GC) were added to the database

${ }^{\mathrm{b}}$ Meals were prepared and analysed for fatty acid composition after formulation
}

\begin{tabular}{|c|c|c|c|c|}
\hline \multirow[t]{2}{*}{ Nutrient } & \multicolumn{2}{|c|}{ Formulated $^{\mathrm{a}}$} & \multicolumn{2}{|l|}{ Analysed $^{\mathrm{b}}$} \\
\hline & Lower fat & Higher fat & Lower fat & Higher fat \\
\hline Protein (\% energy) & 18 & 16 & & \\
\hline Carbohydrates (\% energy) & 59 & 48 & & \\
\hline Dietary fibre (g) & 38 & 31 & & \\
\hline Fat (\% energy) & 23 & 37 & & \\
\hline Saturated fatty acids & 5 & 6 & 4 & 6 \\
\hline 14:0 (myristic acid) & & & 0.3 & 0.5 \\
\hline 16:0 (palmitic acid) & & & 3 & 4 \\
\hline 18:0 (stearic acid) & & & 0.9 & 1.3 \\
\hline Monounsaturated fatty acids & 6 & 20 & 8 & 22 \\
\hline $18: 1$ (oleic acid) & & & 8 & 21 \\
\hline Polyunsaturated fatty acids & 10 & 8 & 11 & 9 \\
\hline 18:2 (linoleic acid) & & & 8 & 7 \\
\hline 18:3 (linolenic acid) & & & 2.3 & 2 \\
\hline Trans fatty acids & $<1$ & $<1$ & 1 & 1 \\
\hline $\mathrm{P}: \mathrm{S}$ & 2 & 1.2 & 2.5 & 1.5 \\
\hline Cholesterol (mg) & 370 & 388 & & \\
\hline
\end{tabular}


observed between patterns of response indicate that a greater proportion of dietary FAs contribute to VLDL-TG in diabetic participants following higher fat intake through chylomicron remnant uptake. However, it is unclear why the oleate concentration was not uniquely elevated (Fig. 3b). This reasoning is speculative, however, because apolipoprotein B48 (apoB48) concentration was not measured in this study. Insulin resistance may result in higher apoB48, but may also increase NEFAs [24] and baseline NEFA concentrations were not different between groups (Table 1). Alternatively, these differences in total and VLDL-TG could result from different methods of quantification, or may indicate that chylomicron remnants were present in the VLDL fraction (supernatant fractions 20400). This is unlikely following a $12 \mathrm{~h}$ fast unless some participants were non-compliant.

Research on lipogenesis usually tests diets that are extreme in form (liquid vs solid, ingested vs feeding tube) or macronutrient content (high simple carbohydrate, carbohydrate overfeeding, very high fat). The current study tested the effects of a mixed diet within usual macronutrient intake ranges. The higher fat concentration was intended to suppress DNL and this effect was observed. Stimulation of hepatic DNL following LF was in agreement with previous research, despite the low glycaemic index/high-fibre content of this diet. The hypothesis that both LF intake and diabetes would result in upregulation of FA synthesis, particularly of palmitic acid, was not observed. The fasting glucose concentration was higher in the diabetes group after LF intake and yet DNL and VLDL-TG concentration were not increased to the extent that they were by this diet in controls. This may indicate sensitivity to LF intake in the control group or an acute metabolic inflexibility in diabetic participants [25].

Research using young normal-weight participants indicates that during fasting, an average of $4.0 \pm 3.6 \%$ of VLDL-TG originated from DNL, whereas $77.2 \pm 14.0 \%$ was from adipose FA release [11]. These estimates of FA synthesis show high variation similar to that found in the current study, but are lower than the control group, which averaged almost $20 \%$ of VLDL-TGFA following LF intake. This could reflect differences in age and BMI, isotope method used or diet design. Indeed, research involving participants with abnormalities in fat metabolism associated with insulin resistance shows that DNL can be elevated in the fasting state. During a high-fat/low-carbohydrate diet, hyperinsulinaemic obese participants had a 3.7-5.3-fold higher rate of DNL than normoinsulinaemic obese or lean participants $(8.5 \pm 0.7 \%$ vs $2.3 \pm 0.3 \%$ vs $1.6 \pm 0.5 \%)$ [26]. After low-fat/high-carbohydrate consumption, DNL was high in all groups, at a rate of approximately 13\% [26]. In patients with non-alcoholic fatty liver disease (NAFLD), the contribution of DNL to VLDL-TG was greater than healthy controls, at rates of $14.9 \pm 2.7 \%$ vs $4.6 \pm 1.1 \%$ [27].
Obese hyperinsulinaemic NAFLD patients exhibit DNL rates of up to $37 \%$ (mean $22.4 \% \pm 8.2 \%$ ) [12]. Indeed, both disease state and diet have important roles in individual lipogenesis rates, with insulin resistance potentially increasing the lipogenic capacity of participants. The present study showed that DNL rates are related to measures of insulin resistance following the LF diet; however, BMI had a stronger relationship to DNL rates following HF. This suggests that the relationship between metabolic measures and clinical indices may depend on dietary factors.

To gain an appreciation for the variability in amount and composition, concentration of de novo synthesised FAs for individual participants is shown (Fig. 4). Participant 13 shows complete suppression of DNL by the HF diet. LF resulted in $69 \mathrm{mg}$ of de novo FA at a synthesis rate of $3 \%$ of total VLDL-TG FA, which is still a small amount of synthesis. Conversely, participant 5 synthesised VLDLTGFA at a rate of $32 \%$ and $24 \%$, resulting in net synthesis of $1925 \mathrm{mg}$ and $739 \mathrm{mg}$, mostly as saturated fatty acids ( $71 \%$ and $81 \%$ of total net de novo FAs) following LF and HF diets, respectively. Hence, there is a potential to modulate FA composition to a more saturated profile because this participant had almost twice the amount of saturated FAs in VLDL-TG following LF than HF diets $(1.51 \mathrm{mmol} / 1$ vs $0.89 \mathrm{mmol} / \mathrm{l})$. This study confirms that palmitate is highly synthesised in humans and may be a useful surrogate for total FA synthesis. It should be noted that the amount varies, as de novo palmitate ranged from $44 \%$ to $84 \%$ of total net de novo FAs (mean $=71 \%$ ).

Two studies have estimated synthesis rates of FAs other than palmitate $[23,28]$. Comparisons with this study are difficult because of vastly different feeding regimens and methods for measuring DNL. Both studies estimate DNL after ingestion of a very-high-carbohydrate liquid meal [23, 28]. Aarsland and Wolfe [23] used an extremely hyperenergetic high-carbohydrate fat-devoid enteral/parenteral feeding design and measured FA synthesis using ${ }^{13} \mathrm{C}$ labelled acetate and mass isotopomer distribution analysis. Synthesis of FAs other than palmitate was not detected in the basal state. However, after hyperalimentation, synthesis rates of oleate and stearate were similar to those in the current study, with greater variation in rates for the five healthy men (palmitate, oleate and stearate synthesis was approximately $45 \%, 22 \%$ and $9 \%$ of the respective FA pools [23]). For the control group in the current study, synthesis rates of palmitate, stearate and oleate were 55\%, $25 \%$ and $6 \%$ of the pool for each FA, respectively. De novo synthesised palmitoleate was $15 \%$ and myristate was $73 \%$ of the total VLDL-TG palmitoleate and myristate pools. Research by Chong et al. used $\mathrm{D}-\left[\mathrm{U}^{13} \mathrm{C}\right]$ fructose or D$\left[\mathrm{U}^{13} \mathrm{C}\right]$ glucose to trace the fate of dietary sugars [28]. This method underestimates FA synthesis; however, relative ${ }^{13} \mathrm{C}$-labelling of some FAs was estimated from the 
supernatant fractions 20-400 from the lipoprotein TG pool. Tenfold as much label from $\left[{ }^{13} \mathrm{C}\right]$ fructose was detected in palmitate than in stearate or myristate [28]. Using ${ }^{2} \mathrm{H}$ incorporation, data from the present study indicate that the amount of palmitate synthesised is more than sixfold that of either oleate, stearate or myristate. The total size of these pools determines the quantitative difference. While myristate synthesis rate is very high at $>50 \%$ of total myristate (Fig. 3c, d), oleate synthesis rate is much lower at $\sim 5 \%$ of total oleate. This translates to similar amounts of these de novo synthesised FAs in VLDL-TG (Fig. 3e, f). It is concluded that in participants who have higher rates of DNL, synthesis of FAs other than palmitate is highly variable but can be quantitatively and qualitatively important.

This is the first research that uses ${ }^{2} \mathrm{H}$ incorporation techniques combined with GC/P/IRMS to determine DNL of individual non-essential FAs. Future research should examine the relationship between FA synthesis rates or composition and the presence of markers or incidence of specific metabolic diseases and phenotypes. Diet is particularly important in light of its significant effects on stimulation or downregulation of FA synthesis and activity of specific elongation/desaturation enzymes. Lipogenesis during the postprandial period must also be considered, as switching sources of FA for VLDL-TG may occur, increasing the fractional contribution of DNL following a meal [11]. The current study had sufficient power to detect differences in FA synthesis rates within and between groups using two-way ANOVA $(n=14)$; however, power decreases when separated into diabetes and control groups $(n=7)$. Although FA synthesis decreased in all but one participant/group following HF, high interindividual response dictates that in order to detect differences between the diets fed, 16 controls and 33 diabetic participants would need to complete the crossover design. Feeding a more lipogenic diet may result in a larger change and smaller numbers required. Further investigation is warranted.

In conclusion, lipogenesis is upregulated in some individuals and produces saturated FAs, potentially affecting overall composition of TG in VLDL. There are unique effects of both diet and diabetes on lipid metabolism. The amount of dietary fat consumed is important in determining changes in DNL. Lower fat intake stimulates FA synthesis rate and increases VLDL-TG FA concentration. Dietary response differed in diabetic participants in this study, perhaps indicating increases in contribution from NEFAs or dietary FAs. Additional analyses will delineate the contribution of NEFAs to VLDLTG composition and DNL changes during the postprandial period. Variations in synthesis of total and saturated FAs in individual participants may be of particular importance.

Acknowledgements We are grateful to the participants who volunteered for this study. We appreciate the technical assistance of O. Levner (IRMS), S. Goertz (GC), A. Goulding (meal preparation) and S. Calder, RN (blood draws). Research was supported through grants from the Canadian Diabetes Association, Canola Council of Canada and Natural Sciences and Engineering Research Council (NSERC) (M. T. Clandinin). M. S. Wilke was partially supported by a NSERC Postgraduate Scholarship. The abstract for this study was presented at the Second World Congress on Controversies to Consensus in Diabetes, Obesity and Hypertension, Barcelona, Spain, 30 October to 2 November 2008 .

Duality of interest The authors declare that there is no duality of interest associated with this manuscript.

\section{References}

1. Parks EJ (2001) Effect of dietary carbohydrate on triglyceride metabolism in humans. J Nutr 131:2772S-2774S

2. Austin MA, Hokanson JE, Edwards KL (1998) Hypertriglyceridemia as a cardiovascular risk factor. Am J Cardiol 81:7B-12B

3. Forsythe CE, Phinney SD, Fernandez ML et al (2008) Comparison of low fat and low carbohydrate diets on circulating fatty acid composition and markers of inflammation. Lipids 43:65-77

4. King IB, Lemaitre RN, Kestin M (2006) Effect of a low-fat diet on fatty acid composition in red cells, plasma phospholipids, and cholesterol esters: investigation of a biomarker of total fat intake. Am J Clin Nutr 83:227-236

5. Raatz SK, Bibus D, Thomas W, Kris-Etherton P (2001) Total fat intake modifies plasma fatty acid composition in humans. J Nutr 131:231-234

6. Layne KS, Goh YK, Jumpsen JA, Ryan EA, Chow P, Clandinin MT (1996) Normal subjects consuming physiological concentrations of 18:3(n-3) and 20:5(n-3) from flaxseed or fish oils have characteristic differences in plasma lipid and lipoprotein fatty acid concentrations. J Nutr 126:2130-2140

7. Field CJ, Angel A, Clandinin MT (1985) Relationship of diet to the fatty acid composition of human adipose tissue structural and stored lipids. Am J Clin Nutr 42:1206-1220

8. Warensjo E, Riserus U, Vessby B (2005) Fatty acid composition of serum lipids predicts the development of the metabolic syndrome in men. Diabetologia 48:1999-2005

9. Clandinin MT, Cheema S, Field CJ, Baracos VE (1993) Dietary lipids influence insulin action. Ann N Y Acad Sci 683:151-163

10. Rivellese AA, Maffettone A, Vessby B et al (2003) Effects of dietary saturated, monounsaturated and n-3 fatty acids on fasting lipoproteins, LDL size and post-prandial lipid metabolism in healthy subjects. Atherosclerosis 167:149-158

11. Barrows BR, Parks EJ (2006) Contributions of different fatty acid sources to very low-density lipoprotein-triacylglycerol in the fasted and fed states. J Clin Endocrinol Metab 91:1446-1452

12. Donnelly KL, Smith CI, Schwarzenberg SJ, Jessurun J, Boldt MD, Parks EJ (2005) Sources of fatty acids stored in liver and secreted via lipoproteins in patients with nonalcoholic fatty liver disease. J Clin Invest 115:1343-1351

13. Heath RB, Karpe F, Milne RW, Burdge GC, Wootton SA, Frayn KN (2003) Selective partitioning of dietary fatty acids into the VLDL TG pool in the early postprandial period. J Lipid Res 44:2065-2072

14. Vedala A, Wang W, Neese RA, Christiansen MP, Hellerstein MK (2006) Delayed secretory pathway contributions to VLDLtriglycerides from plasma NEFA, diet, and de novo lipogenesis in humans. J Lipid Res 47:2562-2574

15. Hudgins LC (2000) Effect of high-carbohydrate feeding on triglyceride and saturated fatty acid synthesis. Proc Soc Exp Biol Med 225:178-183 
16. Aarsland A, Chinkes D, Wolfe RR (1996) Contributions of de novo synthesis of fatty acids to total VLDL-triglyceride secretion during prolonged hyperglycemia/hyperinsulinemia in normal man. J Clin Invest 98:2008-2017

17. Konrad SD, Cook SL, Goh YK, French MA, Clandinin MT (1998) Use of deuterium oxide to measure de novo fatty acid synthesis in normal subjects consuming different dietary fatty acid composition. Biochim Biophys Acta 1393:143-152

18. Canadian Diabetes Association, Clinical Practice Guideline Expert Committee (2003) 2003 clinical practice guidelines for the prevention and management of diabetes in Canada. Can $\mathrm{J}$ Diabetes 27:S1-S152

19. Musliner TA, Long MD, Forte TM et al (1991) Dissociation of high density lipoprotein precursors from apolipoprotein Bcontaining lipoproteins in the presence of unesterified fatty acids and a source of apolipoprotein A-I. J Lipid Res 32:917-933

20. Hargreaves KM, Clandinin MT (1987) Phosphatidylethanolamine methyltransferase: evidence for influence of diet fat on selectivity of substrate for methylation in rat brain synaptic plasma membranes. Biochim Biophys Acta 918:97-105

21. Jones PJ, Winthrop AL, Schoeller DA et al (1988) Evaluation of doubly labeled water for measuring energy expenditure during changing nutrition. Am J Clin Nutr 47:799-804
22. Ebine N, Feng JY, Homma M, Saitoh S, Jones PJ (2000) Total energy expenditure of elite synchronized swimmers measured by the doubly labeled water method. Eur J Appl Physiol 83:1-6

23. Aarsland A, Wolfe RR (1998) Hepatic secretion of VLDL fatty acids during stimulated lipogenesis in men. J Lipid Res 39:12801286

24. Duez H, Pavlic M, Lewis GF (2008) Mechanism of intestinal lipoprotein overproduction in insulin resistant humans. Atheroscler Suppl 9:33-38

25. Parks EJ, Skokan LE, Timlin MT, Dingfelder CS (2008) Dietary sugars stimulate fatty acid synthesis in adults. J Nutr 138:10391046

26. Schwarz JM, Linfoot P, Dare D, Aghajanian K (2003) Hepatic de novo lipogenesis in normoinsulinemic and hyperinsulinemic subjects consuming high-fat, low-carbohydrate and low-fat, high-carbohydrate isoenergetic diets. Am J Clin Nutr 77:43-50

27. Diraison F, Moulin P, Beylot M (2003) Contribution of hepatic de novo lipogenesis and reesterification of plasma non esterified fatty acids to plasma triglyceride synthesis during non-alcoholic fatty liver disease. Diabetes Metab 29:478-485

28. Chong MF, Fielding BA, Frayn KN (2007) Mechanisms for the acute effect of fructose on postprandial lipemia. Am J Clin Nutr 85:1511-1520 